\title{
Immunohistochemical Expression of SOX2 in Benign and Malignant Ovarian Serous Tumors at the Anatomic Pathology Unit H. Adam Malik Hospital Medan
}

\author{
Abdul Hamid Hasan ${ }^{\text {a* }}$, Betty ${ }^{\mathrm{a}}$, Lidya Imelda Laksmia, Soekimin ${ }^{\mathrm{a}}$, Joko S Lukito ${ }^{\mathrm{a}}$ \\ a.Anatomical Pathology Departement, Faculty of Medicine, Universitas Sumatera Utara, Medan, Indonesia \\ hamidhasannas@gmail.com
}

\begin{abstract}
Background: Ovarian neoplasms are most often found in women with an incidence of $80 \%$ of benign tumors and the rest are malignant tumors of the ovary. Ovarian cancer in Indonesia ranks third most cases that occur in women after breast cancer and liver cancer. Serous epithelial ovarian tumors are the most common subtype of ovarian cancer. SOX2 (SRY/sex determining region Y-box2) is a transcription factor that plays a role in cell proliferation and is also a marker of cancer stem cells. The presence of these cancer stem cells will affect the success of therapy because they are resistant to chemotherapy and radiotherapy.

Aim: Knowing how to assess the expression and how the immunohistochemical expression of SOX2 in benign and malignant ovarian serous tumors.

Method: This study is a descriptive analytic study using surgical tissue paraffin block preparations from patients with a diagnosis of benign and malignant ovarian serous tumors in the Anatomical Pathology Unit of HAM Hospital Medan with 39 cases. This research has been approved by the Health Research Ethics Commission, Faculty of Medicine, University of North Sumatra.

Results: Based on the immunohistochemical staining of SOX2, the benign subtype was found to be low in expression (71.4\%), the low-grade subtype to have high expression (18.2\%) and the high-grade subtype to have more high expression (81.8\%).

Conclusion: In this research it was found that the highest expression of SOX2 was found in the benign subtype with low expression, but in the high-grade serous subtype high expression was found.
\end{abstract}

Keywords: ovarian serous tumors; benign and malignant; immunihistochemical staining, SOX2

\section{Introduction}

Ovarian neoplasms are the most common in women with an incidence of $80 \%$ being benign tumors and the rest being malignant ovarian tumors. Ovarian cancer accounts for about 239,000 new cases and 152,000 deaths worldwide every year. ${ }^{1}$ According to GLOBOCAN 2018 data, there were 13,310 (3.8\%) new cases of ovarian cancer in Indonesia, with a mortality rate of 7,842 (3.8\%) and ranks third most cases that occur in women after breast cancer and liver cancer. ${ }^{2}$

Epithelial ovarian tumors are the most common malignant tumors, accounting for $90 \%$ of all cases of ovarian cancer. ${ }^{3}$ These tumors have seven subtypes, namely serous, mucin, endometrioid, clear cell, transitional cell/brenner type, squamous cell tumor and mixed. resembling a variety of cells. Based on the WHO classification, each subtype of ovarian epithelial tumor is further divided into benign, borderline, and malignant tumors. ${ }^{4}$ Serous epithelial ovarian tumors are the most common subtype of ovarian cancer. A number of studies have supported a two-tier scoring system for serous ovarian carcinoma based on histology, 
namely high grade and low grade. ${ }^{5}$

SRY (sex determining region Y)-box 2 (SOX2) is a large SOX family group consisting of transcription factors, important in the regulation of developmental processes and cell type specification. ${ }^{6}$ Since the discovery of the first SOX gene, over the last 20 years 20 genes from the SOX gene family have been identified in mammals and classified into protein specificity groups. ${ }^{7}$

SOX2 is a transcription factor that plays a role in cell proliferation and is also a marker of cancer stem cells. ${ }^{8}$ The presence of cancer stem cells affects the success of therapy, because these stem cells are resistant to chemotherapy and radiotherapy. ${ }^{9}$ The SOX2 gene is located on chromosome $3 \mathrm{q} 26$, a region that is frequently amplified in high grade serous carcinoma. ${ }^{10}$ The presence of SOX2 expression is associated with the prognosis of ovarian serous tumors. ${ }^{11}$ Therefore, the researcher wanted to know the expression and relationship based on SOX2 subtype in histopathological tissue with the diagnosis of ovarian serous tumors in patients at Haji Adam Malik Hospital Medan.

\section{Method}

This study is a descriptive analytic study using paraffin block samples from 39 cases that had been diagnosed histopathologically as benign and malignant ovarian serous tumors. Paraffin blocks were cut and immunohistochemical preparations were made using SOX2 antibody, with an outward pattern on the cell nucleus. The positive control used was lung squamous cell carcinoma. The semi-quantitative assessment of SOX2 expression by the researcher and two specialists in Anatomic Pathology was double-blind. This research has received approval from the Health Research Ethics Commission, Faculty of Medicine, University of North Sumatra with no: 186/TGL/KEPK FK USU-RSUP HAM/2020.

\section{Results}

Out of a total of 39 paraffin block samples diagnosed histopathologically as ovarian serous tumors found the majority aged 50 years with a total of 21 cases (53.8\%). Based on tumor size, the most cases were tumors measuring $>10 \mathrm{~cm}$, namely 18 cases $(46.2 \%)$, followed by tumors measuring $1-5 \mathrm{~cm}$ in 12 cases (30.8\%) and the remaining tumors measuring $>5-10 \mathrm{~cm}$ being in 9 cases $(23.1 \%)$. Based on the location of the tumor, the majority of tumors were unilateral in 25 cases $(64.1 \%)$. Based on histopathological subtypes, 20 cases were found to be benign (51.3\%), high grade cases were 17 cases $(43.6 \%)$ and only 2 cases were low grade $(5.1 \%)$. Based on lymphocyte infiltration / intratumoral TILs, 16 very low cases (41\%), 14 low cases (35.9\%), and 9 high cases $(23.1 \%)$. While lymphocyte infiltration / stromal TILs were found in 15 cases high (38.5\%), 13 cases low (33.3\%), and 11 very low cases (28.2\%). Based on lymphovascular invasion (LVI), the majority were negative in 24 cases $(61.5 \%)$. Meanwhile, based on the expression of SOX2, the majority of cases were low grade, namely 28 cases $(71.8 \%)$ and the remaining 11 cases were high grade $(28.2 \%)$. 
Table 1. Characteristics of patients with ovarian serous tumors

\begin{tabular}{lcc}
\hline Characteristics & Total $(\mathbf{n})$ & Percentage $\mathbf{( \% )}$ \\
\hline Age & & \\
$<50$ years & 18 & 46.2 \\
$\geq 50$ years & 21 & 53.8 \\
Tumor size & & 30.8 \\
$1-5 \mathrm{~cm}$ & 12 & 23.1 \\
$>5-10 \mathrm{~cm}$ & 9 & 46.2 \\
$>10 \mathrm{~cm}$ & 18 & \\
Tumor Location & & 64.1 \\
Unilateral & 25 & 35.9 \\
Bilateral & 14 & \\
Histopathological subtype & & 51.3 \\
Benign & 20 & 5.1 \\
Low grade & 2 & 43.6 \\
High grade & 17 & \\
TILs Intratumoral & & 23.1 \\
High & 9 & 35.9 \\
Low & 14 & 41 \\
Very low & 16 & 38.5 \\
TILs Stromal & & 33.3 \\
High & 15 & 28.2 \\
Low & 13 & 38.5 \\
Very low & 11 & 61.5 \\
LVI & & \\
Positive & 15 & 71.8 \\
Negative & 24 & \\
SOX2 Expression & 11 & \\
High grade & 28 & \\
Low grade & 39 & \\
\hline Total & & \\
\hline
\end{tabular}

In this study, it was found the majority of high expression SOX2 were in the high grade histopathological subtype, as many as 9 cases $(81.8 \%)$ and the remaining 2 cases in the low grade subtype (18.2\%). No high expression of SOX2 was found in the benign subtype. While low expression SOX2 was found in the majority in benign histopathological subtypes, namely 20 cases $(71.4 \%)$ and the remaining 8 cases $(28.6 \%)$ in high grade subtypes. No low expression of SOX2 was found in the low grade subtype.

Table 2. Distribution of SOX2 by Histopathological Subtype

\begin{tabular}{lc|c|c|c}
\hline $\begin{array}{l}\text { Histopathological } \\
\text { Subtype }\end{array}$ & $\begin{array}{c}\text { High } \\
\text { Expression }\end{array}$ & $\boldsymbol{\%}$ & $\begin{array}{c}\text { Low } \\
\text { Expression }\end{array}$ & \% \\
\hline Benign & 0 & 0 & 20 & 71.4 \\
Low grade & 2 & 18.2 & 0 & 0 \\
High grade & 9 & 81.8 & 8 & 28.6 \\
\hline
\end{tabular}




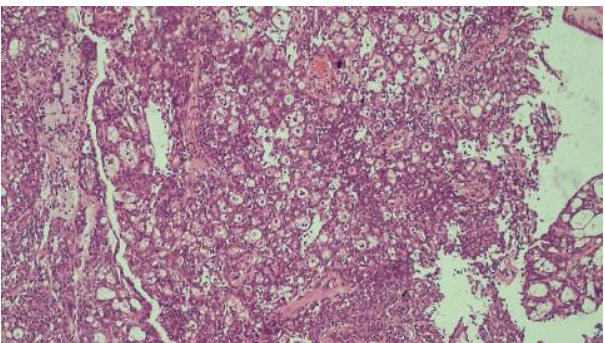

TILs intratumoral High (HE 100x)

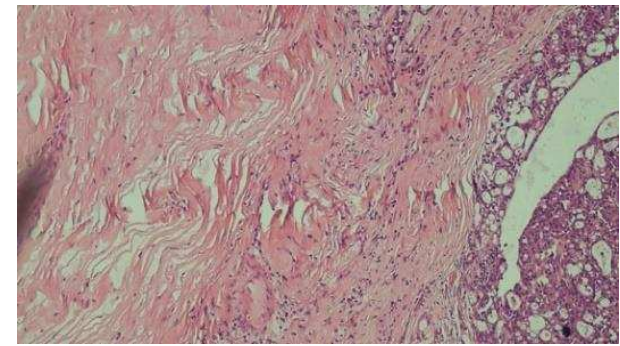

TILs stroma very low (HE 100x)

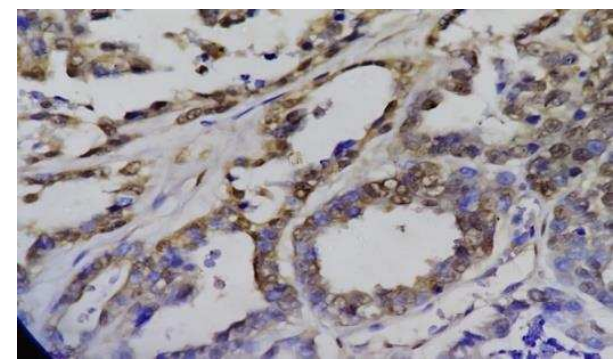

SOX2 high expression (IHC 400x)

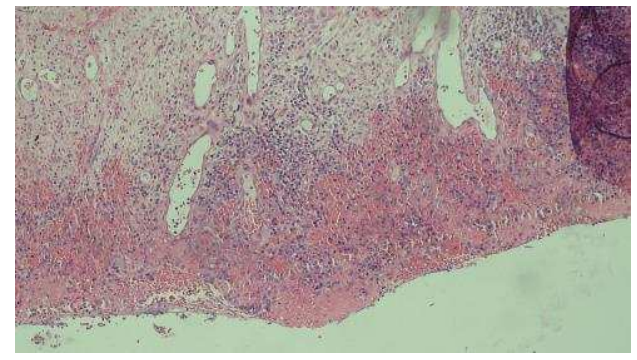

TILs stroma Low (HE 100x)

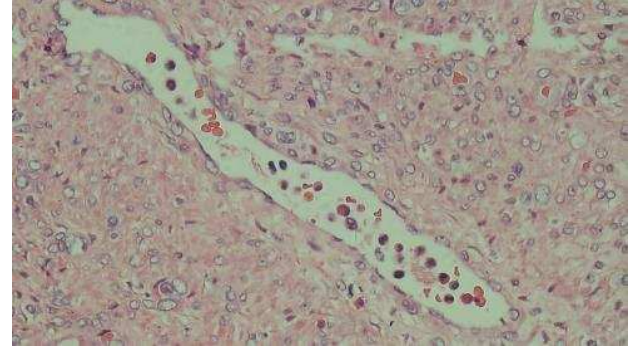

LVI positive (HE 400x)

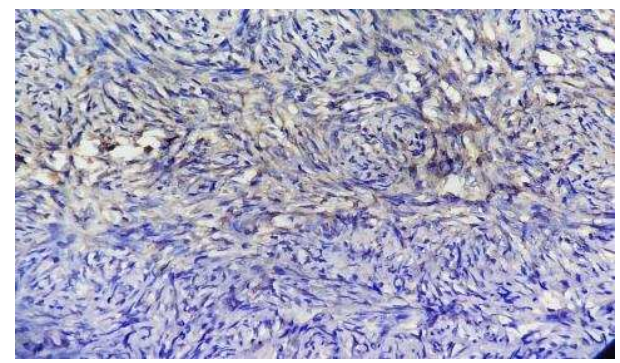

SOX2 low expression (IHC 400x)

\section{Discussion}

In this study, it was found that most patients were aged 50 years than those aged $<50$ years. This study is in line with the research of Chen et al., 2015 which found patients aged 50 years as many as 342 cases $(69.5 \%)$ out of 492 patients with epithelial ovarian carcinoma. ${ }^{12}$ In this study, the majority of patients with ovarian serous tumors were women older than 50 years (post menopause). Researchers suspect this is closely related to increased levels of the hormones Follicle Stimulating Hormone (FSH), Luteinizing Hormone ( $\mathrm{LH})$ and Gonatropin Horrmone $(\mathrm{GH})$ in postmenopausal women. The increase in these hormones is known to trigger carcinogenesis, tumor progression and inhibit apoptosis of ovarian serous carcinoma cells. ${ }^{13}$

Tumor size in this study was taken based on WHO Female and Genitalia in 2014 where in this study the largest size was found to be $>10 \mathrm{~cm}$. Research Gockley et al. in 2017 found the most serous tumor size at $>5 \mathrm{~cm}$ in as many as 65 cases $(47.8 \%) .{ }^{14}$ Researchers suspect that tumor growth in the early stages is rarely 
detected. However, as time goes on, the tumor gets bigger and causes clinical manifestations and that's when the patient begins to have a self-examination and the diagnosis of serous ovarian tumor is established.

Based on the subtype, ovarian serous carcinoma was found mostly in the benign subtype, as many as 20 cases $(51.3 \%)$. This is in accordance with the research of Harahap et al. who reported that the most cases found in H. Adam Malik Hospital Medan were benign subtypes, namely 68 cases $(26.6 \%) .{ }^{15}$ This is also in accordance with the study of Vivien et al., who reported that serous tumors with benign subtype most commonly occurred in the fourth and fifth decades and in this study as many as 18 cases (46.2\%) were found at the age $<50$ years. ${ }^{16}$

TILs is a protective immune system that can inhibit tumor growth. The higher the TILs found, the higher the grade of the tumor. Research by James et al. in 2017 who found the most stromal TILs and intratumoral TILs at very low, 122 cases (39\%) and 13 cases (57\%). The study explained that an increase in the grade of intratumoral and stromal TILs was associated with a better prognosis, but that a significant association was only found in intratumoral TILs. ${ }^{17}$ This is different from the TILs in this study assessed on the basis of intratumoral and stromal where the most intratumoral TILs were found at very low and the most stromal TILs were found at high (Table 1). In this study, different results were found between stromal TILs and intratumoral TILs. Researchers suspect this is because most of the samples obtained with a diagnosis of benign histopathological subtype.

LVI in this study found more negative than positive (Table 1). Research by Chen et al., 2015 found that from 334 cases with high grade serous carcinoma, there were more positive LVI cases, namely 224 cases $(67.1 \%)$ than negative cases, namely 110 cases $(32.9 \%)$, while from 22 low grade cases, Serous carcinomas had more negative LVI, namely 16 cases $(72.7 \%)$ than positive ones, namely 6 cases $(27.3 \%)$. According to Chen et al. LVI in ovarian carcinoma is associated with aggressive tumors, including advanced serous tumors, high-grade serous carcinomas, high-grade ovarian epithelial carcinomas and lymph node metastases. LVI tumor is an important predictor of progression-free and overall survival in early-stage patients, but not in advanced-stage patients. ${ }^{12}$ The negative LVI that was often found in this study was probably due to the fact that the most samples obtained were benign histopathological subtypes.

SOX2 expression can be expressed in normal conditions, but in malignancy can occur over expression. increased expression of SOX2 correlated the degree of malignancy and tumor progression. In this study, it was found that all cases of benign serous tumors had low SOX2 expression and low grade serous ovarian tumors had high expression. However, in contrast to high grade serous ovarian tumors, they showed dualism of expression, where the highest expression of SOX2 was found, followed by low expression of SOX2 (Table 2). This is in line with the research of Pham et al. who found 95 cases with high SOX2 expression in high grade serous ovarian tumors, followed by 25 low SOX2 expression. ${ }^{18}$ It is not known exactly why this phenomenon occurs. However, it is suspected that the regulation of tumor cell signaling may vary, especially in high grade serous ovarian tumors. Further research is needed to study the mechanism.

\section{Conclusion}

In this study, serous ovarian tumors were found to be more common in patients with ovarian serous tumors aged 50 years than those aged $<50$ years, the most tumor size was $>10 \mathrm{~cm}$, unilateral ovarian tumors were more common than bilateral and the most subtypes were benign. TILs were assessed on the basis of intratumoral and stromal where the most intratumoral TILs were at very low while the most stromal TILs 
were at high and LVI was negative. The highest expression of SOX2 was found in the benign subtype with low expression, but the high-grade serous subtype found high expression.

\section{Acknowledgements}

We acknowledge to all staff and resident of Anatomical Pathology Departement of Universitas Sumatera Utara, H.Adam Malik Hospital, Medan, Indonesia for all is help and cooperation.

\section{Ethical Approval} study.

The Health Reserch Ethical Committee, Universitas Sumatera Utara, Medan, Indonesia approved this

\section{References}

1. Ferlay J, Soerjomataram I, Ervik M, Dikshit R, Eser S, Mathers C, et al. GLOBOCAN 2012 v1.0, Cancer Incidence and Mortality Worldwide: IARC CancerBase No. 11. Lyon, France: International Agency for Research on Cancer, 2013[2016-09-09].

2. Ferlay J, Colombet M, Soerjomataram I, Mathers C, Parkin DM, Pineros M, et al.. Estimating the global cancer incidence and mortality in 2018: GLOBOCAN source and methods. International Journal of Cancer. 2019; pp.1941-1953.

3. Screening for Ovarian Cancer, Daniel L. Clarke-Pearson, MD The new england journal of medicine. N Engl J Med 2009;361: pp.170-177

4. Longacre TA, Wells M. Serous tumors. In: Kurman RJ, Carcangiu ML, Harrington CS, Young RH, editors. WHO Classification of Tumors of Female Reproductive Organs 4th ed. Lyon: IARC. 2014; pp.10-15.

5. Kurman RJ, Shih IM. The dualistic model of ovarian carcinogenesis: Revised, revised and expanded. Am J Pathology. 2016; 186: pp.733-747

6. Singer G, Kurman RJ, Chang HW, Cho SK, Shih IeM. Diverse tumorigenic pathways in ovarian serous carcinoma. Am J Pathol. 2002; 160: pp.1223-1228. [PubMed: 11943707]

7. Sieben NL, Macropoulos P, Roemen GM, Kolkman-Uljee SM, Jan Fleuren G, Houmadi R, et al.. In ovarian neoplasms, BRAF, but not KRAS, mutations are restricted to low grade serous tumours. J Pathol. 2004; 202: pp.336-340. [PubMed: 14991899]

8. Kohler MF, Marks JR, Wiseman RW, Jacobs IJ, Davidoff AM, Clarke-Pearson DL, et al.. Spectrum of mutation and frequency of allelic deletion of the p53 gene in ovarian cancer. J Natl Cancer Inst. 1993; 85: pp.1513-1519. [PubMed: 8360934]

9. Salani R, Kurman RJ, Giuntoli R 2nd, Gardner G, Bristow R, Wang TL, et al.. Assessment of TP53 mutation using purified tissue samples of ovarian serous carcinomas reveals a much higher mutation rate than previously reported and does not correlate with drug resistance. Int J Gynecol Cancer. 2008; 18: pp.487-491. [PubMed: 17692090]

10. Staebler A, Karberg B, Behm J, Kuhlmann P, Neubert U, Schmidt H, et al.. Chromosomal losses of regions on 5q and lack of highlevel amplifications at 8q24 are associated with favorable prognosis for ovarian serous carcinoma. Genes Chromosomes Cancer 2006; 45: pp. 905-17.

11. Castillo SD \& Sanchez-Cespedes M. The SOX family of genes in cancer development: Biological relevance and opportunities for therapy. Expert Opin Ther Targets 16: pp. 903-919, 2012.

12. Chen M, Jin Y, Bi Y, Li Y, Shan Y, Pan L. Prognostic Significance of Lymphovascular Space Invasion in Epithelial Ovarian Cancer. Journal of Cancer 2015, Vol. 6. 2015; 6(5): pp. 412-419.

13. Zhang Z, Zhu Y, Lai Y, Wu X, Feng Z, Yu Y, et al. Follicle-stimulating hormone inhibits apoptosis in ovarian cancer cells by regulating the OCT4 stem cell signaling pathway. INTERNATIONAL JOURNAL OF ONCOLOGY 43: pp.1194-1204, 2013.

14. Gockley A, Melamed A, Bregar AJ, Clemmer JT, Birrer M, Schorge O, et al. Prognostic Significance of Lymphovascular Space Invasion in Epithelial Ovarian Cancer. Gynecol Obstetrics. 2017 March; 129(3): pp.439-447.

15. Harahap FP, Edianto D, Sahil MF. Siregar MFG, Tala MRZ, Luther D. Profile of Epithelial Ovarian Cancer Based on Histologic Type, Staging, Type of Treatment and 5 year Survival Rate On Adam Malik General Hospital Medan 2008-2012. USU repository. 2013; pp.1-4

16. Chen VW, Ruiz B, Killen JL, Cote TR, Wu XC, Correa CN, et al. Pathology and classification of ovarian tumors.Cancer Volume 97, Issue S10, 2003. pp.2631-2642

17. James FR, Linan MJ, Alsop J, Mack M, Song H, Brenton JD, et al. Association between tumor infiltrating lymphocytes, histotype and clinical outcome in epithelial ovarian cancer. BMC Cancer (2017) 17:657.

18. Pham DL, Scheble V, Bareiss P, Fischer A, Beschorner C, Adam A, et al. SOX2 expression and prognostic significance in ovarian carcinoma. Int J Gynecol Pathol. 2013 Jul;32(4): pp.358-67. 


\section{Author Details}

First Author - dr. Abdul Hamid Hasan, resident of Anatomical Pathology Departement. Faculty of medicine, Universitas Sumatera Utara, Medan, Indonesia, id email: hamidhasannas@gmail.com

Second Author - Dr. dr. Betty, M.Ked(PA), Sp.PA(K), Lecture and Staff Anatomical Pathology Departement. Faculty of medicine, Universitas Sumatera Utara, Medan, Indonesia

Third Author - Dr. dr. Lidya Imelda Laksmi, M.Ked(PA), Sp.PA, Lecture and Staff Anatomical Pathology Departement. Faculty of medicine, Universitas Sumatera Utara, Medan, Indonesia

Fourth Author - dr. Soekimin, Sp.PA(K), Lecture and Staff Anatomical Pathology Departement. Faculty of medicine, Universitas Sumatera Utara, Medan, Indonesia

Fifth Author - dr. Joko S Lukito, Sp.PA(K), Lecture and Staff Anatomical Pathology Departement. Faculty of medicine, Universitas Sumatera Utara, Medan, Indonesia.

Corresponding Author - Abdul Hamid Hasan, resident of Anatomical Pathology Departement. Faculty of medicine, Universitas Sumatera Utara, jl. Universitas No.1 gedung Abdul Hakim, Medan, Indonesia, E-mail ID: hamidhasannas@gmail.com 